\title{
A Definition of the Magnetic Transition Temperature Using Valence Bond Theory
}

\author{
Joaquim Jornet-Somoza, ${ }^{*, \dagger}, \ddagger$ Mercè Deumal, ${ }^{\dagger}$ Juan Borge, ${ }^{\ddagger}$ and Michael A. Robb $₫$ \\ $\dagger$ Departament de Ciència de Materials i Química Física and IQTCUB, Facultat de \\ Química, Universitat de Barcelona, Martí i Franquès 1, E-08028 Barcelona \\ $\ddagger$ Nano-Bio Spectroscopy Group and ETSF Scientific Development Centre, Department of \\ Materials Physics, University of the Basque Country, CFM CSIC-UPV/EHU-MPC and \\ DIPC, Tolosa Hiribidea 72, E-20018 Donostia-San Sebastián \\ IDepartment of Chemistry, Imperial College London, South Kensington Campus, SW \\ 2AZ London
}

E-mail: j.jornet.somoza@gmail.com 


\begin{abstract}
Macroscopic magnetic properties are analyzed using Valence Bond theory. Commonly the critical temperature $T_{C}$ for magnetic systems is associated with a maximum in the energy-based heat capacity $C_{p}(T)$. Here a more broadly applicable definition of the magnetic transition temperature $T_{C}$ is described using spin moment expectation value (i.e. applying the spin exchange density operator) instead of energy. Namely, the magnetic capacity $C_{s}(T)$ reflects variation in the spin multiplicity as a function of temperature, which is shown to be related to $\partial[\chi T(T)] / \partial T$. Magnetic capacity $C_{s}(T)$ depends on long-range spin interactions that are not relevant in the energy-based heat capacity $C_{p}(T)$. Differences between $C_{s}(T)$ and $C_{p}(T)$ are shown to be due to spin order/disorder within the crystal, that can be monitored via a Valence Bond analysis of the corresponding magnetic wavefunction. Indeed the concept of the Boltzmann spin-alignment order is used to provide information about the spin correlation between magnetic units. As a final illustration, the critical temperature is derived from the magnetic capacity for several molecular magnets presenting different magnetic topologies that have been experimentally studied. A systematic shift between the transition temperatures associated with $C_{s}(T)$ and $C_{p}(T)$ is observed. It is demonstrated that this shift can be attributed to the loss of long-range spin correlation. This suggests that the magnetic capacity $C_{s}(T)$ can be used as a predictive tool for the magnetic topology, and thus for the synthetic chemists.
\end{abstract}




\section{Introduction}

The first attempts to understand magnetism at the atomic level were carried out by Langevin and Weiss using semiempirical theories. In 1905, Langevin ${ }^{1,2}$ provided a good description of paramagnetism for an atom or molecular magnet that carried a permanent magnetic moment, whose spatial distribution was to be given by the Boltzmann factor. In 1907, Weiss ${ }^{3}$ predicted the existence of the Curie point $\left(T_{C}\right)$ below which ferromagnetism sets in by means of introducing the effect of an internal or molecular field proportional to the intensity of magnetization. Later on, in 1913, the angular momentum was quantized and, hence, the magnetic moment of an atom or a molecule-based magnet using Bohr's quantum theory, ${ }^{4}$ which employed classical mechanics combined with quantum conditions. This resulting in the definition of the magnetic susceptibility expressed by the Langevin-Debye formula,

$$
\chi(T)=\frac{N_{A} \mu_{B}^{2}}{3 k_{B} T} \mu_{e f f}^{2}
$$

where $\mu_{\text {eff }}$ and $\mu_{B}$ is the effective magnetic moment and Bohr magneton respectively, $N_{A}$ is the Avogadro number, $k_{B}$ is the Boltzmann constant, and $T$ the temperature of the system. In 1927, Van Vleck ${ }^{5}$ established the general formula for the magnetic susceptibility using quantum mechanics for the zero external magnetic field limiting case. Van Vleck also introduced the temperature dependence of the magnetic moment $(\mu) .{ }^{5}$ For the computation of $\mu$ it was necessary to know the energy of all stationary states of the system, $E_{n}$, to compute the magnetic susceptibility. With the quantum derivation of Van Vleck, the magnetism of many atoms, molecules and compounds could be well described and their magnetic behavior could be also characterized as antiferromagnetic (AFM), ferromagnetic (FM) or diamagnetic, as a function of the microscopic magnetic interaction $\left(J_{i j}\right)$ between the spins $i$ and $j$. The $J_{i j}$ values are obtained from fitting the magnetic susceptibility curve to a given model ${ }^{6}$ or computed using quantum chemistry tools. ${ }^{7,8}$

The magnetic behavior of a molecule-based crystal depends on the relative arrangement of 
the spin-carrying units within the crystal. The relative alignment of two interacting $S=1 / 2$ radicals generates three cases: (1) a FM interaction, when the spins of both radicals are coupled parallel to generate a triplet; (2) an AFM interaction, when the adjacent spins are coupled antiparallel to form a singlet; and (3) a paramagnetic behavior, when the unpaired spins show no magnetic interaction between them. In the case of many centers, the FM and AFM cases give rise to two limiting cases: (1) all adjacent spins are parallel to each other (which generates a bulk ferromagnet, when the FM interactions extend over the three directions of space), and (2) all adjacent spins are antiparallel (which results in a bulk antiferromagnet, when the AFM interactions extend over the three directions of space). Note that a particular case of antiferromagnetism is diamagnetism. Diamagnetism results from antiparallel spin-pairing with no net spin momentum. In between these two extreme cases, there are other possible combinations, which include dominant FM or AFM behavior, such as metamagnetism, change in magnetic dimensionality, or spin-canting ${ }^{6}$

The formulation of the macroscopic magnetic properties including magnetic susceptibility $\chi(T)$, magnetization $M(H)$, and heat capacity $C_{p}(T)$ can be formulated with quantum mechanics using statistical mechanics. ${ }^{5}$ However, the definition of the critical temperature $T_{C}$ below which the magnetic systems order and behave as FM, AFM, etc. is not completely clear. A maximum in the heat capacity $C_{p}(T)$ is usually taken to indicate a second order phase transition. ${ }^{9,10}$ However, this transition does not correspond to the appropriate magnetic order/disorder physical transition, since it is associated with changes in energy, and not with changes in spin. Thus it is not the most suitable critical temperature for magnetism.

In 1962, Fisher ${ }^{11}$ suggested that there was a correlation between a $C_{p}(T)$ and $\partial[\chi T(T)] / \partial T$, implying that both had a similar physical interpretation. ${ }^{12-15}$ In this paper, we propose a definition of the magnetic critical temperature derived in the same way as $C_{p}(T)$, but using the spin moment rather than the energy. We shall refer to this as the magnetic capacity $C_{s}(T) . C_{s}(T)$ is defined so that it accounts for the variation of the mean value of the spin multiplicity, for the overall system, as a function of temperature. The quantity $\partial[\chi T(T)] / \partial T$ 
can be obtained directly from experimental data and is proportional to the magnetic capacity, $C_{s}(T)$. With these definitions we can identify long-range order or the lack of it with those cases where $T_{C}$ obtained from either $\partial[\chi T(T)] / \partial T$ or $C_{s}(T)$ agrees or not with $T_{C}$ defined in terms of $C_{p}(T)$.

In order to develop the main ideas related to the magnetic capacity $C_{s}(T)$, we will present the mathematical expressions for macroscopic magnetic properties using Valence Bond theory. We show that the spin exchange density matrix operator in Valence Bond theory, which describes the bonding interaction, is also useful for the microscopic interpretation of the different magnetic observables. The expressions of $C_{s}(T)$ and $C_{p}(T)$ will be given using spin exchange density matrix elements, $P_{i j} \cdot{ }^{16,17}$ It will be shown that the heat capacity $C_{p}(T)$ involves the derivative of the energy, whereas $C_{s}(T)$ involves changes on the spin moment.

The theoretical development and discussion of this paper is structured as follows. We start with the usual derivation of the macroscopic magnetic properties from statistical mechanics, and then present these very same magnetic properties using the spin exchange density matrix elements $P_{i j}$. This gives rise to the definition of the magnetic capacity, $C_{s}(T)$. The physical insight from this reformulation will then be discussed. Next we will discuss the interpretation of the magnetic capacity, in terms of the Valence Bond magnetic wavefunction, using the exchange density matrix ( $P_{i j}$ terms). This reformulation allows the interpretation of magnetic phenomena in terms of short- and long-range spin couplings. ${ }^{18}$ Such short- and long-range effects explain the differences between $C_{p}(T)$ and $\partial[\chi T(T)] / \partial T$. Finally, we will illustrate the application of the definition of $T_{C}$ defined in terms of $C_{s}(T)$ for the description of magnetic critical temperatures of some molecular magnets that have been characterized experimentally. 


\section{Theory}

In this section, we first derive the magnetic properties $\chi(T), C_{p}(T)$ and $C_{s}(T)$ using a description of the magnetic wavefunction obtained from Valence Bond theory. This in turn allows the definition of the Boltzmann magnetic spin-alignment order $\left(\tilde{\mu}_{i j}\right)$, which describes the temperature dependent spin-correlation between two radical centers $(i$ and $j)$. Then, an illustrative example is presented using a model linear system consisting in 4 spin-carrying $S=$ $1 / 2$ radicals with nearest-neighbor magnetic interactions (hereafter $4 \mathrm{~s}$ model). In general, a 'ns' model stands for the number 'n' of radicals in the model and for 's' $S=1 / 2$ unpaired spins.

Within the framework of molecule-based magnets, the magnetic susceptibility, $\chi$, and heat capacity, $C_{p}$, are the macroscopic magnetic properties defining the temperature at which the magnetic second order phase transition occurs, i.e. the magnetic transition temperature. Equations (2) and (3) show the theoretical expressions for the magnetic susceptibility $\chi(T)$, and heat capacity $C_{p}(T)$ in absence of orbital angular momentum, developed by Van Vleck in terms of the properties of each microstate,

$$
\begin{gathered}
\chi(T)=\frac{N_{A} g^{2} \mu_{B}^{2}}{3 k_{B} T} \sum_{n} \frac{S_{n}\left(S_{n}+1\right)\left(2 S_{n}+1\right) e^{-E_{n} / k_{B} T}}{\sum_{n^{\prime}}\left(2 S_{n^{\prime}}+1\right) e^{-E_{n^{\prime}} / k_{B} T}} \\
C_{p}(T)=\frac{N_{A}}{k_{B} T^{2}}\left\{\frac{\sum_{n} E_{n}^{2}\left(2 S_{n}+1\right) e^{-E_{n} / k_{B} T}}{\sum_{n^{\prime}}\left(2 S_{n^{\prime}}+1\right) e^{-E_{n^{\prime}} / k_{B} T}}-\frac{\left[\sum_{n} E_{n}\left(2 S_{n}+1\right) e^{-E_{n} / k_{B} T}\right]^{2}}{\left[\sum_{n^{\prime}}\left(2 S_{n^{\prime}}+1\right) e^{-E_{n^{\prime}} / k_{B} T}\right]^{2}}\right\}
\end{gathered}
$$

where $g$ and $\mu_{B}$ are the gyromagnetic constant and Bohr magneton respectively, and $E_{n}$ and $S_{n}$ are the energy and spin multiplicity of the $n$ microstate. From these expressions, we can see that the energy and spin multiplicity of the $n$ microstates are the only quantities that depend directly on the system and give the magnetic properties in equations (2) and (3).

In previous work, we have shown that these magnetic microstates can be obtained by solving an algebraic eigenvalue problem associated with a Heisenberg Hamiltonian for spin $1 / 2$ particles, ${ }^{19}$ 


$$
\hat{H}=-2 \sum_{i, j} J_{i j} \hat{S}_{i} \hat{S}_{j}
$$

where $\hat{S}_{i}$ is the spin operator acting on center $i$, and $J_{i j}$ is the microscopic magnetic interaction between spin centers $i$ and $j$. As written, the Hamiltonian acts on a space of neutral covalent Slater determinants where each orbital $i$ occurs only once with either $\alpha$ or $\beta$ spin. Thus the operators $\hat{S}_{i}$ operate on the spin of orbital $i$. The $J_{i j}$ are the exchange integrals of Heitler-London Valence Bond theory (for a model two-electron system with orbitals $i$ and $j$ ). The $J_{i j}$ can be obtained from ab initio computations on a pair of radicals. ${ }^{20}$ See Supporting Information Section 1 for a discussion on the working strategy followed to bring together $J_{i j}$ and macroscopic magnetic properties.

Equation (4) corresponds to a model Hamiltonian with computed parameters that reduces the problem of chemical binding to coupling of electron spins. Anderson ${ }^{21}$ was the first to recognize that Heisenberg Hamiltonians might be understood as effective Hamiltonians computed from an exact full configuration interaction (CI) Hamiltonian using a model space of neutral Valence Bond determinants formed from $n$ electrons in $n$ atomic orbitals. The use of such spaces in quantum chemistry was first proposed in this context by Malrieu and co-workers. ${ }^{16,22-25}$

Our implementation of a Heisenberg Hamiltonian model has been used to study magnetic interactions in many problems. ${ }^{26-32}$ In order to keep this paper self-contained, before reformulating equations (2) and (3), we will briefly review some essential aspects of the Heisenberg spin Hamiltonian (equation 4) and how it can be used to study magnetic properties of molecule-based magnets.

Traditionally, Valence Bond theory, formulated as a Heisenberg Spin Hamiltonian, is based on the use of localized active orbitals. One uses a neutral covalent determinant basis $\left[\Phi_{K}, \ldots, \Phi_{L}\right]$ in which each orbital occurs at most once with $\alpha$ or $\beta$ spin (see Supporting Information Section 2 for an example of the construction of a basis set consisting in 4 
electrons). The orbitals are assumed to be non-orthogonal of the Coulson-Fischer type. ${ }^{33,34}$ However, the orthogonality is reflected on the parametrization of the $J_{i j}$ and the orbitals are never treated explicitly. ${ }^{35}$

Lets now define the spin-exchange operator $\hat{P}_{i j}$ as permutation operator that interchange the spin coordinate of two electrons. For example, the application of the spin-exchange operator over four 2 electrons spin primitive functions, leads to:

$$
\begin{aligned}
& \hat{P}_{12} \alpha(1) \alpha(2)=\alpha(1) \alpha(2) \\
& \hat{P}_{12} \alpha(1) \beta(2)=\beta(1) \alpha(2) \\
& \hat{P}_{12} \beta(1) \alpha(2)=\alpha(1) \beta(2) \\
& \hat{P}_{12} \beta(1) \beta(2)=\beta(1) \beta(2)
\end{aligned}
$$

Then, we can construct a certain operator using the spin permutation that has the same effect as the spin operator product $\left(\hat{S}_{i} \cdot \hat{S}_{j}\right)$ presents in the Heisenberg Hamiltonian (equation 4). This is the so called Dirac identity, ${ }^{17}$ which in the case of two spins is

$$
[\hat{S}(1) \hat{S}(2)] \Theta(1,2)=-\left(\frac{1}{2} \hat{P}_{12}-\frac{1}{4} \hat{I}\right) \Theta(1,2)
$$

i.e.

$$
P_{12}=\left\langle-\left(2[\hat{S}(1) \hat{S}(2)]+\frac{1}{2} \hat{I}\right)\right\rangle
$$

where $\Theta(1,2)$ is any two-electron spin function, and $\hat{I}$ is the identity operator. The spinexchange density matrix $P_{i j}$ is a useful analytic concept, which matrix elements are computed as the expectation values of $\hat{P}_{i j}$ (equation 7). These can be defined, using the general expression of a two electron operator in second quantization, ${ }^{36}$ as

$$
\left\langle\Psi\left|\hat{P}_{i j}\right| \Psi\right\rangle=-\sum_{i, j, k, l}\left\langle i(1) j(2)\left|\hat{S}(1) \hat{S}(2)+\frac{1}{4} \hat{I}(1,2)\right| k(1) l(2)\right\rangle \times\left\langle\Psi\left|a_{i}^{\dagger} a_{j}^{\dagger} a_{l} a_{k}\right| \Psi\right\rangle
$$

where $a_{i}^{\dagger}, a_{i}$ are the creation and annihilation operator acting over the $i$ spin-orbital. The 
integration of the spatial part gives Kronecker- $\delta$ terms, and by resolving the spin operator using step-up and step-down operators, we obtain the final expression: ${ }^{37}$

$$
\begin{gathered}
P_{i j}=P_{i j}^{\alpha \beta}-P_{i j}^{\alpha \alpha} \\
P_{i j}^{\alpha \beta}=\frac{1}{2}\left\langle\Psi\left|a_{i \alpha}^{\dagger} a_{j \alpha} a_{j \beta}^{\dagger} a_{i \beta}+a_{i \beta}^{\dagger} a_{j \beta} a_{j \alpha}^{\dagger} a_{i \alpha}\right| \Psi\right\rangle \\
P_{i j}^{\alpha \alpha}=\frac{1}{2}\left\langle\Psi\left|a_{i \alpha}^{\dagger} a_{j \alpha}^{\dagger} a_{j \alpha} a_{i \alpha}+a_{i \beta}^{\dagger} a_{j \beta}^{\dagger} a_{j \beta} a_{i \beta}\right| \Psi\right\rangle
\end{gathered}
$$

One can see that the exchange density matrix $P_{i j}$ elements are the difference between exchange terms for electrons of different $P_{i j}^{\alpha \beta}$ and same $P_{i j}^{\alpha \alpha}$ spin, respectively. In general $P_{i j}$ can be obtained in terms of the coefficients of the expansion of the multiconfigurational magnetic wavefunction, as:

$$
\begin{aligned}
P_{i j}^{\alpha \beta} & =\frac{1}{2} \sum_{K, L} c_{K} c_{L}\left\langle\Phi_{K}\left|a_{i \alpha}^{\dagger} a_{j \alpha} a_{j \beta}^{\dagger} a_{i \beta}\right| \Phi_{L}\right\rangle \\
P_{i j}^{\alpha \alpha} & =\frac{1}{2} \sum_{K} c_{K}^{2}\left\langle\Phi_{K}\left|a_{i \alpha}^{\dagger} a_{j \alpha}^{\dagger} a_{j \alpha} a_{i \alpha}\right| \Phi_{K}\right\rangle
\end{aligned}
$$

The $P_{i j}$ terms are just a two-particle density matrix elements that can be obtained from any CI computation. ${ }^{37}$ For wavefunctions composed of several Valence Bond structures, the values of $P_{i j}$ give a quantitative measure of the bonding, as suggested early by Penney and Moffitt. ${ }^{38}$ Further, the spin-exchange density matrix elements are related to the total spin by: ${ }^{17}$

$$
S(S+1)=-\frac{N(N-4)}{4}-\sum_{i<j} P_{i j}
$$

where $N$ is the number of unpaired electrons. Notice that equation (11) can be used to compute the spin multiplicity of each of the multiconfigurational eigenfunctions of the Heisenberg Hamiltonian.

Then, the general spin-exchange Heisenberg Hamiltonian (equation 4) can now be written 
as: ${ }^{18}$

$$
\hat{H}=-\sum_{i j} J_{i j}\left(2 \hat{S}_{i} \hat{S}_{j}+\frac{1}{2} \hat{I}\right)=\sum_{i j} J_{i j} \hat{P}_{i j}
$$

where the expectation values of both Hamiltonians (equations 4 and 12) are related by a shift due to the identity operator. Further, one can see that the energy spectrum can be also obtained using,

$$
\Delta E_{n}=\sum_{i<j} J_{i j} \Delta P_{i j}^{n},
$$

where $\Delta E_{n}=E_{n}-E_{0}$ and $\Delta P_{i j}^{n}$ refer to the differences of the energy values and $P_{i j}$ matrix elements between the $n^{\text {th }}$ excited state and the ground state, respectively.

As mentioned above, the exchange density matrix elements $P_{i j}$ are related to the relative alignment of the spin of electrons in magnetic centers $i$ and $j$. For a two-electron system $(N=$ 2, 2s model) with spins either coupled antiparallel or parallel or uncoupled, the reference $P_{i j}$ values are given in Figure 1. For $S=0$ (Figure 1a), $\langle\hat{S}(i) \hat{S}(j)\rangle=-3 / 4$ and the $P_{i j}$ element has a value of +1.0 , while if coupled parallel $S=1$ (Figure 1b), $\langle\hat{S}(i) \hat{S}(j)\rangle=+1 / 4$ and the $P_{i j}$ will be -1.0 . In addition, for uncoupled spins, $\left\langle S^{2}\right\rangle=2\left[\frac{1}{2}\left(\frac{1}{2}+1\right)\right]=+3 / 2$ and $\langle\hat{S}(i) \hat{S}(j)\rangle=0$, the value of the corresponding $P_{i j}$ element is -0.5 (see Figure 1c). For that reason, from a VB perspective, $P_{i j}$ can be suitably denoted as magnetic bond-order $(\mu$ BO). We have given some discussion of physical interpretation of the spin exchange density matrix elements in the Supporting Information Section 3. There we give the $P_{i j}$ for the Kekulé and Dewar VB structures of the $\pi$-electrons of the benzene molecule.

We now discuss the temperature dependence into the magnetic properties using statistical mechanics of the canonical ensemble. In such scheme, the population of each microstate $(n)$ is defined by the Boltzmann distribution function, $f_{B}^{n}(T)=\frac{\left(2 S_{n}+1\right)}{Z} e^{-E_{n} / k_{B} T}$, where $Z$ stands for the canonical partition function and the numerator stands for the spin degeneracy of 


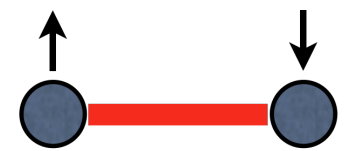

(a) antiparallel spin alignment $P_{i j}=+1$

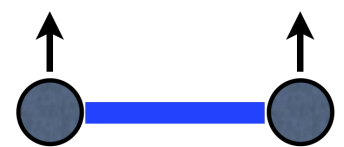

(b) parallel spin alignment $P_{i j}=-1$

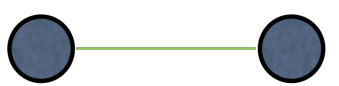

(c) non-ordered spin alignment $P_{i j}=-0.5$

Figure 1: Relative alignment of the spin of electrons in magnetic centers $i$ and $j$ for a twoelectron system (2s): (a) antiparallel, (b) parallel, and (c) non-ordered or uncoupled. The $P_{i j}$ values are also given from equation (11).

the microstate $n$. Therefore, the temperature dependent expectation values of the spin multiplicity and energy can now be expressed in terms of the eigenvalues of the Heisenberg Hamiltonian equation (12), i.e. the spin multiplicity and energy of each microstate. Using equations (11) and (13), these $\langle E\rangle_{T}$ and $\left\langle S^{2}\right\rangle_{T}$ expectation values can be expressed in terms of the spin exchange density matrix elements,

$$
\begin{gathered}
\langle E\rangle_{T}=\sum_{n} \Delta E_{n} \cdot f_{B}^{n}(T)=\sum_{n} \sum_{i<j} J_{i j} \Delta P_{i j}^{n} \cdot f_{B}^{n}(T), \\
\left\langle S^{2}\right\rangle_{T}=\sum_{n} S_{n}\left(S_{n}+1\right) \cdot f_{B}^{n}(T)=-\frac{N /(N-4)}{4}-\sum_{n} \sum_{i<j} P_{i j}^{n} \cdot f_{B}^{n}(T),
\end{gathered}
$$

Now we can define the temperature dependent spin-alignment order matrix, $\tilde{\boldsymbol{\mu}}(T)$. The elements of $\tilde{\boldsymbol{\mu}}(T)$ between two spin carrying units $i$ and $j$ are defined as the Boltzmann weighted spin exchange density matrix element,

$$
\tilde{\mu}_{i j}(T)=\sum_{n} P_{i j}^{n} \cdot f_{B}^{n}(T)
$$

This Boltzmann weighted magnetic bond-order matrix carries information about the correlated alignment between all the spin units and its temperature behavior. We shall refer to $\tilde{\boldsymbol{\mu}}(T)$ (equation 16) as Boltzmann magnetic $\mu$-bond order. The macroscopic magnetic properties, in terms of the spin exchange density matrix elements, $P_{i j}$, of each microstate now arise as follows. In order to obtain the expression for the heat capacity $C_{p}(T)$ as a 
function of the temperature, we differentiate $\langle E\rangle_{T}$ (equation 14) with respect to temperature. Thus the magnetic capacity, $C_{s}(T)$, is obtained by differentiating $\left\langle S^{2}\right\rangle_{T}$ (equation 15) with respect to temperature,

$$
\begin{aligned}
& C_{p}(T)=\frac{\partial\langle E\rangle_{T}}{\partial T}=\frac{N_{A}}{k_{B} T}\left\{\sum_{n}\left[\left(\sum_{i<j}\left(J_{i j} \cdot P_{i j}^{n}(J)\right)\right) f_{B}^{n}(T)\left[\sum_{m}\left(E_{m}-E_{n}\right) f_{B}^{m}(T)\right]\right]\right\}, \quad(17) \\
& C_{s}(T)=\frac{\partial\left\langle S^{2}\right\rangle_{T}}{\partial T}=\frac{N_{A}}{k_{B} T}\left\{\sum_{n}\left[\left(\sum_{i<j}\left(P_{i j}^{n}(J)+P_{i j}^{n}(0)\right)\right) f_{B}^{n}(T)\left[\sum_{m}\left(E_{m}-E_{n}\right) f_{B}^{m}(T)\right]\right]\right\} .
\end{aligned}
$$

In equations (17) and (18), we have identified two types of spin-exchange density matrix elements, $P_{i j}(J)$ and $P_{i j}(0)$, which occur in the Heisenberg spin Hamiltonian energy expression (equation 12). $\quad P_{i j}(J)$ corresponds to a $P_{i j}$ where the value of $J_{i j}$ is finite, while $P_{i j}(0)$ corresponds to a $P_{i j}$ where the value of $J_{i j}$ is vanishingly small (in previous calculations it was defined to be $\left.<|0.05| \mathrm{cm}^{-1}\right) .{ }^{19,39}$ Thus $P_{i j}(0)$ corresponds to the longrange spin coupling that has no energetic effect in the Heisenberg Hamiltonian. Thus the $P_{i j}(J)$ (and $\tilde{\mu}_{i j}(J, T)$ ) elements are those where magnetic interaction $J_{i j}$ has a non-negligible value, i.e. a connected/short-range spin alignment is present, while the terms labeled $P_{i j}(0)$ (and $\tilde{\mu}_{i j}(0, T)$ ) have $J_{i j}=0$ (hence it does not appear in equation (17)), and show a nonbonded/long-range spin alignment. This classification turns out to be useful for understanding the physical phenomena that take place upon magnetic transition.

We now discuss some conceptual aspects of the two magnetic properties defined in equations (17) and (18). The heat capacity $C_{p}(T)$ involves a change in the energy of the whole system, and depends on the spin-alignment between the magnetic centers with non-negligible magnetic interactions, $P_{i j}(J)$. The magnetic capacity $C_{s}(T)$ involves a change of the spin moment of the whole system, and depends on the spin-alignment between all pairs of magnetic centers, including those for which $J_{i j}$ is zero. This latter term includes the effect of long-range spin ordering, as experimentally ${ }^{40,41}$ and theoretically ${ }^{18,42}$ suggested. The role of the Boltzmann magnetic $\mu$-bond order and its relation with the spin-exchange density 
matrix values $P_{i j}$ in equations (17) and (18) allows a simple relationship between structure and magnetism. In addition, the maximum of the magnetic capacity function indicates an inflection point at which the long-range spin pairs start to be ordered when cooling from high to low temperature. In contrast, the maximum of the heat capacity describes the transition at which the nearest-spins become correlated, i.e. the existence of a short-range spin ordering.

\section{Results and Discussion}

\section{Conceptual Development}

In this section we shall give some physical insight into the concepts presented in the previous section. This discussion has two facets. On the one hand we wish to demonstrate the effect of $P_{i j}(J)$ and $P_{i j}(0)$ on $C_{s}(T)$ and $C_{p}(T)$ for a model 4 electron system $(4 \mathrm{~s})$. Then we shall discuss the relationship of the experimental quantity $(\partial[\chi T(T)] / \partial T)$ to either $C_{s}(T)$ or $C_{p}(T)$, and analyze the effect of the magnetic topology.

We now illustrate the theoretical ideas just discussed using the Boltzmann magnetic $\mu$ bond order for a four $S=1 / 2$ radical system whose four unpaired electrons interact linearly (4s). The simplest magnetic model results from the interaction between two radical centers with one unpaired electron each (hereafter $2 \mathrm{~s}$ model). However, this model is not adequate to illustrate the different physical meaning of the heat and the magnetic capacities since it does not have any long-range order $P_{i j}(0)$. Thus the simplest model we can use is formed by four $S=1 / 2$ magnetic units linearly interacting $(4 \mathrm{~s})$. We also choose $J=-50 \mathrm{~cm}^{-1}$. We now illustrate the application of the magnetic wave function analysis in terms of $P_{i j}$ using the 4s AFM linear model (see Figure 2a for magnetic topology; and Figures 2b,c for short- and long-range spin alignment, respectively). As we will see bellow, the physical interpretation does not depend on the value of the magnetic interaction, but on the magnetic topology.

The construction and diagonalization of the Heisenberg Hamiltonian (equation 12) al- 
(a)

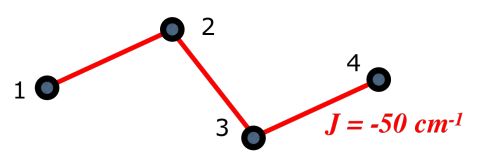

(b)

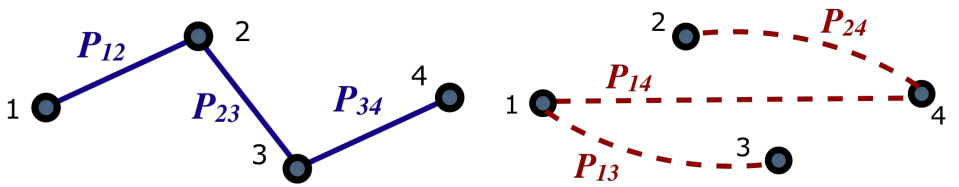

Figure 2: (a) Magnetic topology for a four-spin center 4s antiferromagnetic AFM linear model $\left(J=-50 \mathrm{~cm}^{-1}\right.$ represented as straight red lines). (b) Schematic representation of the short-range spin exchange density matrix elements $P_{i j}(J)$ (solid blue lines). (c) Schematic representation of the long-range spin exchange density matrix elements $P_{i j}(0)$ (dashed brown lines).

lows one to obtain the multiconfigurational magnetic wavefunctions of the ground and excited states of our system (the authors strongly encourage interested readers to address to Supporting Information Section 4 for further details on the chemical analysis of the spin interactions using VB theory). Then, by applying equation (10), the magnetic bond order ( $\mu$-BO) for each spin-pair in each magnetic state can be computed. The individual computed $P_{i j}$ will differ from the ideal values shown in Figure 1 due to the multiconfigurational nature of the magnetic wave function.

The $\mu$-BO (i.e. the exchange density matrix element $P_{i j}$ ) is indicative of the nature of the relative alignment of the spin of electrons in magnetic centers $i$ and $j$ (see Figure 1 for a twoelectron system). Then extending this idea by applying the corresponding Boltzmann factor, we can visualize the temperature evolution of the magnetic $\mu$-bonds as shown in Figure 3a. In other words, the Boltzmann weighted $P_{i j}$ elements enable one to study the temperature dependence of the magnetic $\mu$-bond order, $\tilde{\mu}_{i j}(T)$. Note that parallel spin alignment has been assigned to $P_{i j}$ values ranging from -0.5 to -1.0 , which are displayed in blue in Figure 3a. The antiparallel spins are attributed to $P_{i j}$ from -0.5 to +1.0 (shown in red in Figure 3a). The strength of the $\tilde{\mu}_{i j}(T)$ representation increases (indicated by line thickness) as the temperature decreases. This directly translates into an increase of the spin alignment correlation. Between 53-100 K it is possible to observe that the short-range spin ordering $\tilde{\mu}_{i j}(J, T)$ (i.e. $\left.\left\langle P_{i j}(J)\right\rangle_{T}\right)$ contribution is stronger, as shown by the thickness of the lines in 
(a)

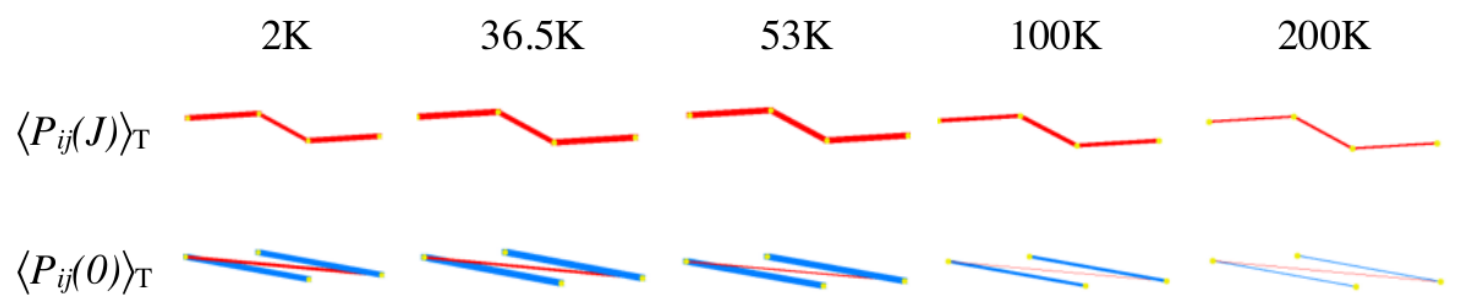

(b)

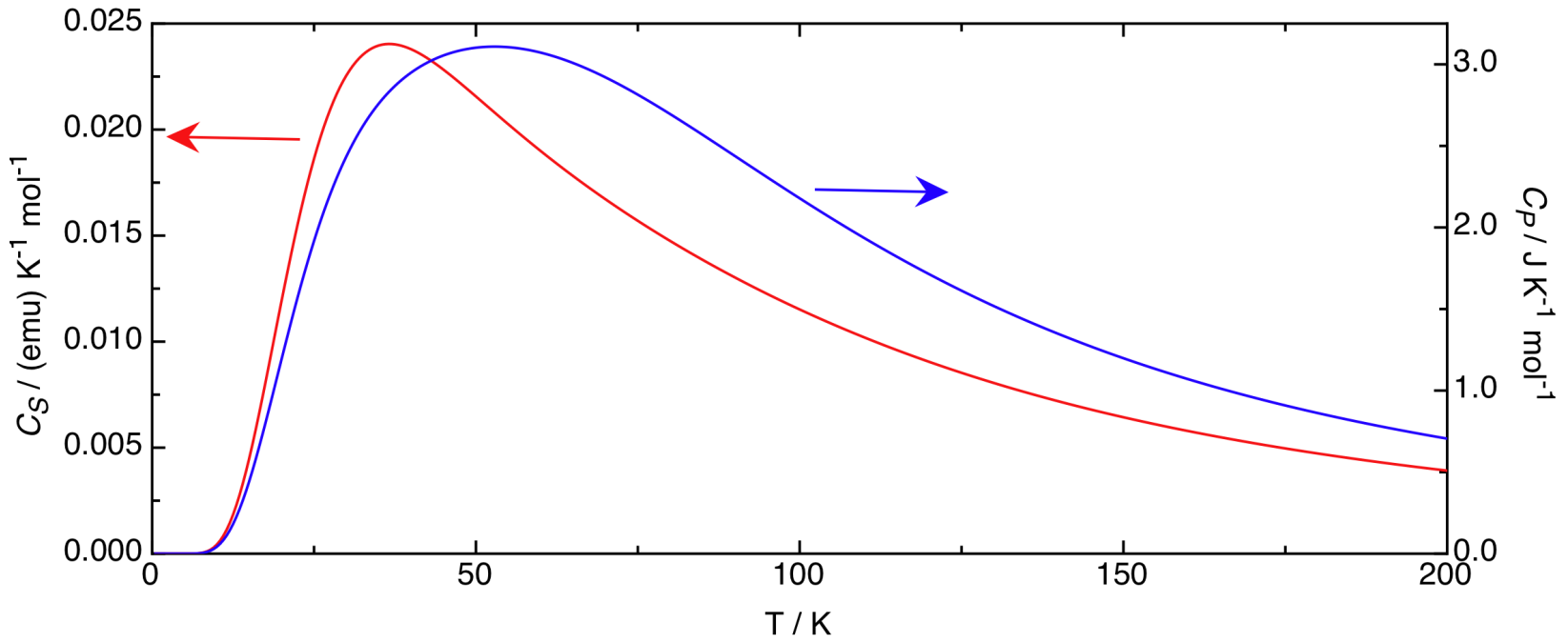

Figure 3: (a) Representation of $\tilde{\mu}_{i j}(J, T)$ (upper) and $\tilde{\mu}_{i j}(0, T)$ (lower) contributions at a temperature of interest for the $4 \mathrm{~s}$ linear AFM system. Notice that parallel (antiparallel) spin alignment is displayed in blue (red). (b) Representation of the magnetic capacity $\left(C_{s}(T)\right.$, red line) and heat capacity $\left(C_{p}(T)\right.$, blue line). All results refer to the 4 s linear AFM system. 
Figure 3a. This in turn correlates with the maximum in $C_{p}(T)$ shown in Figure 3b. For the long-range spin ordering or correlation $\tilde{\mu}_{i j}(0, T)$ (i.e. $\left.\left\langle P_{i j}(0)\right\rangle_{T}\right)$, the actual strengthening takes place between $36.5-53 \mathrm{~K}$, leading to the maximum in $C_{s}(T)$ shown in Figure $3 \mathrm{~b}$. From Figure $3 \mathrm{~b}$ one can see that, when cooling the system from high temperatures, the heat capacity $C_{p}$ begins to increase at higher temperatures than the magnetic capacity $C_{s}$. If one continues lowering the temperature, the maximum of the heat capacity can be observed around 53K. The magnetic capacity curve however displays its maximum value at ca. $36.5 \mathrm{~K}$.

The central idea here is that the largest change of the spin moment of the whole system is accomplished at lower temperatures than the largest change of the energy of the whole system. In the following, we will explain the reason for this shift by simply using the definition of the $\tilde{\mu}_{i j}(T)$, i.e. the fact that the new reformulation (equations 17 and 18) implies Boltzmann factor weighted $P_{i j}$ terms.

$$
\sum_{i j} P_{i j}^{n}(0) \multimap-\sum_{i j} P_{i j}^{n}(J) \multimap
$$

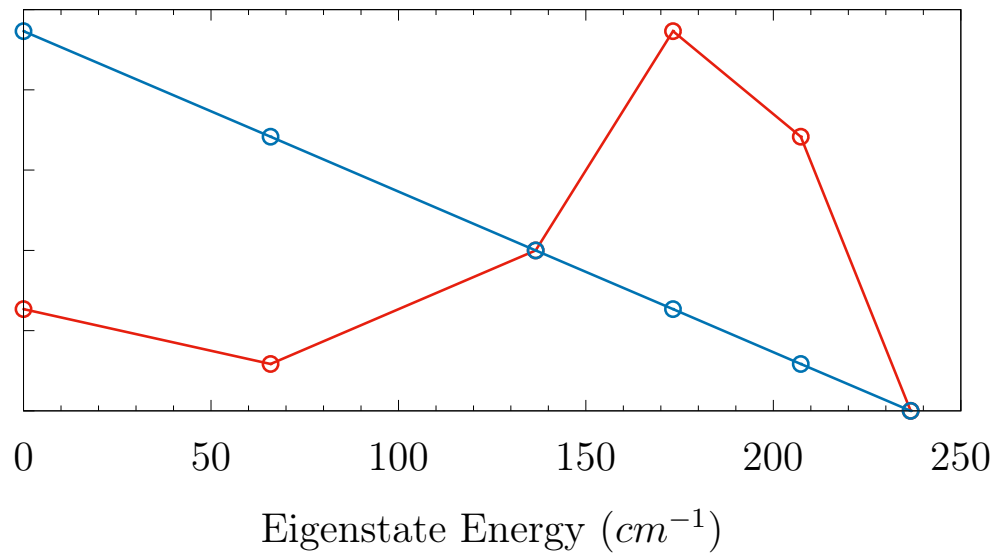

Figure 4: Variation of the sum of spin-exchange density matrix elements $\left(P_{i j}\right)$ as a function of the energy level for a $4 \mathrm{~s}$ linear AFM chain. The different trend of all long-range $P_{i j}(0)$ terms (red, sum over the $P_{i j}(0)$ elements) and short-range $P_{i j}(J)$ terms (blue, sum over the $P_{i j}(J)$ elements) is represented for the six possible eigenstates.

From expressions (17) and (18), it is apparent that the different behavior of $C_{s}(T)$ and $C_{p}(T)$ is directly related to the different $P_{i j}$ contributions (summation over the $i, j$ spin pairs). Figure 4 shows the variation of the sum of the $P_{i j}(0)$ and $P_{i j}(J)$ elements for each magnetic microstate (red and blue lines, respectively). As expected from equation (13), 
we see that the sum of all short-range $P_{i j}(J)$ values linearly decreases as the energy of the eigenstates increases, with slope equal to the magnetic interaction, $J_{i j}$. In contrast, the sum of all long-range $P_{i j}(0)$ terms changes/fluctuates between different states. Now, let us take a look at equations (17) and (18). We can see that $C_{p}(T)$ is proportional to the sum of the $J$-scaled $P_{i j}(J)$ elements while $C_{s}(T)$ is proportional to the sum of all raw $P_{i j}$ elements. This is the key-factor that governs the two different magnetic phase transitions.

The physical interpretation of this fact is easy to explain. By increasing the temperature, the magnetic excited states start to be populated and, in turn, all of the $P_{i j}$ values of each state contribute on the magnetic ordering/disordering (see Supporting Information Section 4 for magnetic states). Since the long-range $\mu$-bond orders, $P_{i j}(0)$, differ significantly between excited states (Figure 4), the global long-range spin ordering tends to cancel out at lower temperatures. In other words, this description enables us to identify two physical processes: while $C_{p}(T)$ measures the short-range spin correlation, $C_{s}(T)$ will give information about long-range correlation. As we shall presently discuss, when the above Boltzmann $\mu$-bond order study is extended to real molecular magnets the conclusions reached were the same as for the 4s AFM chain model (see discussion on KAXHAS in Supporting Information Section $5)$.

Both $C_{p}(T)$ and $C_{s}(T)$ macroscopic properties allow the identification of a characteristic temperature $\left(T_{C}\right)$ at which the system rapidly changes its behavior. In other words, for both descriptions there exists a temperature at which the derivative of the energy or spin moment becomes zero, revealing critical phenomena. We have illustrated the different nature of the transition signaled by the heat capacity and magnetic capacity, $C_{p}(T)$ and $C_{s}(T)$ respectively. Equations (17) and (18) show that the heat capacity $C_{p}(T)$ depends on the variation of the $P_{i j}(J)$ (short-range spin alignment), while the variation of both $P_{i j}(J)$ and $P_{i j}(0)$ (shortand long- range spin alignment, respectively) terms play an important role in determining the magnetic capacity $C_{s}(T)$ (see Figure 5 ). Note that all the analyses here performed have been done using $|0.05| \mathrm{cm}^{-1}$ as cut-off for $J_{i j}$ to differentiate between $P_{i j}(J)$ and $P_{i j}(0)$. Our 
conclusions are however not sensitive to the selected cut-off: (i) $C_{s}(T)$ would not be affected, since it already considers all spin correlations; and (ii) $C_{p}(T)$ would be the property affected, but since it is scaled by $J_{i j}$ itself it already accounts for the threshold chosen.

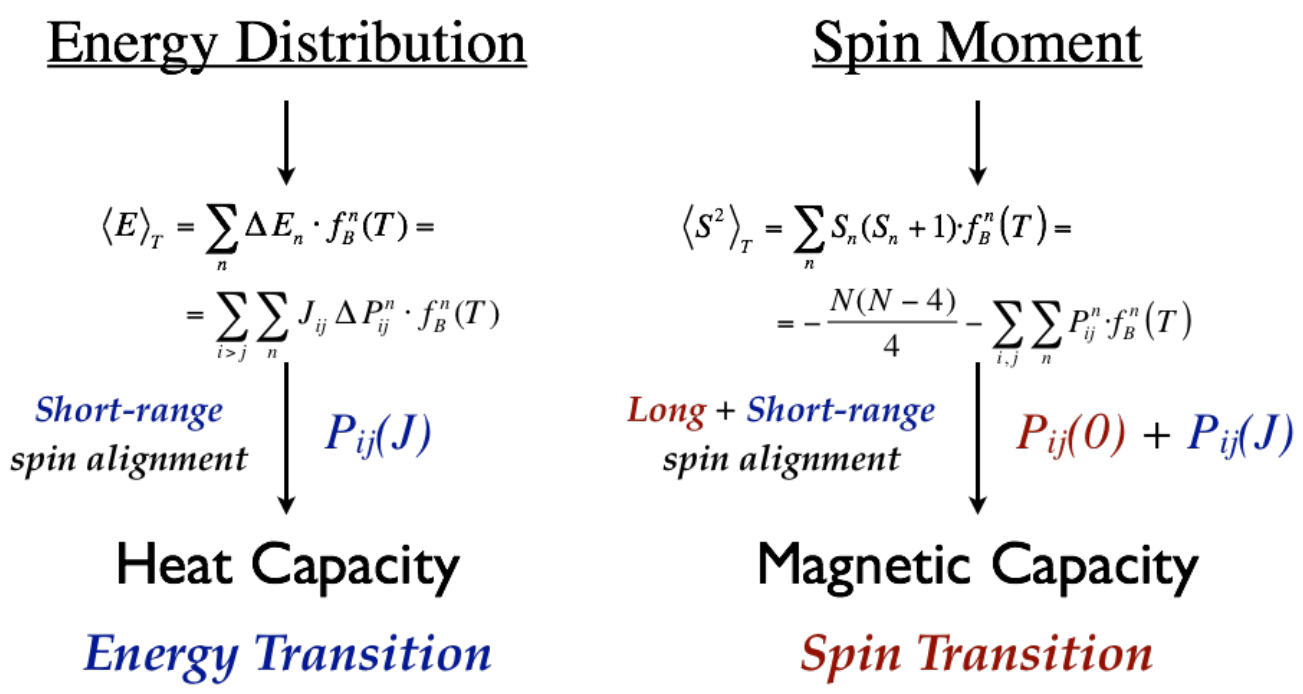

Figure 5: Flowchart connecting the heat capacity $C_{p}(T)$ with its source, which is the energy distribution. Similarly, the magnetic capacity $C_{s}(T)$ is related to the spin moment.

As reported above, the magnetic capacity always reflects a critical temperature $\left(T_{C}^{\mu}\right)$ which is lower to the one found by the heat capacity $\left(T_{C}^{h}\right)$. This is due to the fact that, for extended systems, and due to a thermal mixture of the magnetic microstates, the longrange magnetic ordering is always lost at lower temperatures than the short-range magnetic ordering. This statement demonstrates the complementarity role of both properties in order to properly characterize a magnetic material. Therefore, we can conclude that $T_{C}$ calculated from either $C_{p}(T)$ or $C_{s}(T)$ will be nearly equal only in those molecule-based systems in which the long- range spin correlation can be neglected, or there is no 3D propagation of the spin coupling.

Usually the magnetic transition is characterized following measurements of the heat capacity. ${ }^{10,12-15}$ However, it is often seen that this magnetic critical temperature can also be obtained measuring the first derivative of the magnetic susceptibility times the temperature $(\partial[\chi T(T)] / \partial T)$, which is known as Fisher's heat capacity. ${ }^{12-15}$ This name is adopted in refer- 
ence to the direct proportionality suggested by Fisher, ${ }^{11}$ and used to determine the magnetic ordering. ${ }^{43,44}$

We now discuss the relationship with Fisher's heat capacity. If we compare the new magnetic capacity $C_{s}(T)$, formulated in equation (18), with the well-known expression (1) for the magnetic susceptibility $\chi(T)$, we notice that the effective magnetic moment $\mu_{e f f}^{2}$ can be replaced by:

$$
\chi(T)=\frac{N_{A} \mu_{B}^{2}}{3 k_{B} T} \mu_{e f f}^{2}=\frac{N_{A} g^{2} \mu_{B}^{2}}{3 k_{B} T}\left\langle S^{2}\right\rangle_{T}
$$

Notice that for a non-interacting spin system, whose simplest realization would involve a large energy separation between ground state and first excited states, the Boltzmann distribution can be neglected, and the effective magnetic moment $\left(\mu_{e f f}\right)$ can be considered temperature independent. However, equation (19) shows that temperature dependence has to be taken into account for general cases.

In this context it is clear that what we have defined as magnetic capacity $C_{s}(T)$ ) (equation 18 ) is directly proportional to the derivative of the magnetic susceptibility or Fisher's heat capacity,

$$
\frac{\partial[\chi T(T)]}{\partial T}=\frac{N_{A} g^{2} \mu_{B}^{2}}{3 k_{B}} C_{s}(T)
$$

As we have mentioned above, M.E. Fisher suggested a direct proportionality between $C_{p}(T)$ and $\chi(T)$ observables, giving rise to the fact that both properties should exhibit the same transition temperature. However, our derivation indicates that this proportionality is only accomplished when the magnetic crystal acts as a sum of isolated spin-pairs. In other words, for magnetic materials that can present long-range spin ordering, the critical temperature is no longer correlated with the maximum in $C_{p}(T)$. Thus the magnetic capacity is capable of identifying short- or long-range magnetic $\left(\mu^{-}\right)$bond order or magnetic correlation, which can easily be experimentally measured with the maximum in $\partial[\chi T(T)] / \partial T$. Therefore, one can look in detail at the results of the calculations and understand the nature of the long/short range magnetic order in the crystal. 


\section{Computed $T_{C}$ for several examples using $C_{s}(T)$ and $C_{p}(T)$ and com- parison with experimental maximum in $(\partial[\chi T(T)] / \partial T)$.}

We now test the validity of the $\mu$-bond order concept in magnetic structure calculations via the computation of $T_{C}$ for several examples using $C_{s}(T)$ and $C_{p}(T)$ and comparison with experimental maximum in $(\partial[\chi T(T)] / \partial T)$. For simplicity we will use magnetic units carrying spin $1 / 2$ on a number of key prototypical molecular magnets (see column 1 in Table 1). ${ }^{19,45-50}$ The analyzed molecular magnets are made by $\mathrm{Cu}(\mathrm{II})$-based radicals, except

for UBUBIC and KAXHAS, whose magnetism is due to purely organic radicals. ${ }^{51}$ For all these molecule-based magnets, good agreement between computed and experimental data was obtained for $\chi(T)$ magnetic susceptibility and $C_{p}(T)$ heat capacity temperature response curves using expressions (2) and (3), respectively (see Supporting Information Section 1 for more details on the first principles simulation working strategy). A variety of real magnetic topologies have been examined (column 2 in Table 1), including regular and alternating 1D chains, strong-rail and strong-rung spin-ladders, 2D AFM layer, 3D AFM interacting honeycomb layers and 3D FM diamond-type (see column 3 in Table 1 for most significant calculated $J_{i j}$ values). ${ }^{19,45-50}$

The maximum of $\partial[\chi T(T)] / \partial T$, the so called Fisher's heat capacity, has also been obtained by numerical differentiation from experimental data (see column 4 for $T_{C}^{\partial[\chi T(T)] / \partial T}$ in Table 1$)$. The critical temperature of the magnetic capacity $C_{s}(T)$, namely $T_{C}^{\mu}$, of seven real systems has been calculated applying equation (18) (see column 5 in Table 1). Finally, simulated and experimental values for the critical temperature $T_{C}^{h}$ referring to $C_{p}(T)$, i.e. the temperature at which the magnetic property exhibits a maximum, are compared (see column 6 for $\left.T_{C}^{h}\right)$.

The value of the maximum in $\partial[\chi T(T)] / \partial T$ is the experimental reference point. The value of $T_{C}$ computed from $C_{s}(T)$ is in acceptable agreement with this value $\left(T_{C}^{\mu} \approx T_{C}^{\partial[\chi T(T)] / \partial T}\right)$, 
Table 1: Comparison between computed characteristic temperatures for seven studied prototypical crystals. CCDC name is given in column 1. Magnetic topology and significant $J_{i j}$ values (in $\mathrm{cm}^{-1}$ are given in columns 2 and 3. Characteristic temperatures (in $\mathbf{K}$ ) extracted from $\partial[\chi T(T)] / \partial T$, magnetic capacity, and heat capacity are given in columns 4-6. a \% computed at calculated $T_{C}$ as $\left[T_{C}^{h}\left(C_{p}\right)-T_{C}^{\mu}\left(C_{s}\right)\right] / T_{C}^{h}\left(C_{p}\right) * 100$. Note that Alt=Alternating; $\mathbf{S P}=$ spin-ladder; $\exp =$ experimental; calc $=$ calculated.

\begin{tabular}{lllllll}
\hline $\begin{array}{l}\text { CCDC } \\
\text { name }\end{array}$ & $\begin{array}{l}\text { magnetic } \\
\text { topology }\end{array}$ & $\begin{array}{l}J_{i j} / \mathrm{cm}^{-1} \\
(\text { calc })\end{array}$ & $\begin{array}{l}T_{c}^{\partial[\chi T] / \partial T} \\
(\exp )\end{array}$ & $\begin{array}{l}T_{c}^{\mu}\left(C_{s}\right) \\
(\text { calc })\end{array}$ & $\begin{array}{l}T_{c}^{e}\left(C_{p}\right) \\
(\exp / \text { calc })\end{array}$ & $\begin{array}{l}\%\left(C_{s} / C_{p}\right) \\
(\text { calc })^{a}\end{array}$ \\
\hline & & & & & & \\
CUPYAR $^{45}$ & 1D Chain & -5.23 & 4.31 & 5.60 & $5.22 / 6.85$ & 18.2 \\
UBUBIC $^{46}$ & 1D Alt Chain & $-54.43 /-55.97$ & 74 & 60.20 & $-/ 72.60$ & 17.1 \\
TEXDEG $^{47}$ & Strong-rail SP & $-7.79 /-3.49$ & 6.50 & 6.35 & $-/ 9.20$ & 31.0 \\
XANKED $^{48}$ & Strong-rung SP & $-13.82 /-10.26$ & 35.92 & 47.70 & $-/ 50.10$ & 4.8 \\
CUPYZP $^{49}$ & 2D Layer & -5.62 & 8.4 & 11.65 & $-/ 15.30$ & 23.9 \\
GIVZOB $^{50}$ & 3D Honeycomb & $-8.21,-4.91 /-2.44$ & 6.1 & 6.40 & $-/ 7.75$ & 17.4 \\
KAXHAS $^{19}$ & 3D Bulk FM & $+0.68 /+0.18$ & - & 0.23 & $0.60 / 0.70$ & 67.1 \\
& & & & & & \\
\hline
\end{tabular}

as expected from their direct relationship (see equation 20). The transition temperature $T_{C}^{\mu}$ is thus the most meaningful $T_{C}$ because it results from a magnetic spin transition, i.e. from the thermal variation of the spin multiplicity of the system. $T_{C}^{\mu}$ reflects the importance of the non-connected spin alignment and the dominant effect of long-range spin correlation. The difference between experimental and computed values has been demonstrated to be mainly due to the variation of the crystal structure owed to the temperature at which it has been determined. ${ }^{52}$ Further, if experimental $T_{C}^{\partial[\chi T(T)] / \partial T}$ and calculated $T_{C}^{\mu}$ values are very different it could also be an indication of the presence of impurities in the sample, the existence of different crystal phases, or the simple presence of unanticipated magnetic interactions because we make no assumptions in the simulation of $\chi(T)$.

The non-constancy of the ratio between calculated $C_{s}$ magnetic capacity and calculated $C_{p}$ heat capacity (see column 7 in Table 1) supports that there is no direct proportionality between $\partial[\chi T(T)] / \partial T$ and $C_{p}(T)$. Therefore, Fisher's suggestion is not applicable on real molecular magnets. Interestingly, the difference between transition temperatures appears 
to be extremely dependent on the magnetic topology of each molecular material. Therefore, although this dependence is not the focus of this paper, this fact can be useful for the experimental material science community to predict the magnetic dimensionality of a molecule-based magnet of interest, and then use the appropriate analytical expressions to obtain a fitted $J$ parameter. The calculation of $T_{C}^{\mu}\left(C_{s}\right)$ will thus enable one to estimate the magnetic topology, and hence predict which is the best procedure to perform measurements as a function of the working temperature range, etc.

Finally, comparison between $T_{C}$ computed from $C_{s}(T)$ and $C_{p}(T)$ (both calculated theoretically) should give an indication of the effects of long-range order (indicated numerically (\%) in column 7, Table 1). KAXHAS, which is a 3D bulk ferromagnet, has the largest $\left(\% T_{C} C_{s} / C_{p}\right)$ value. This can be expected, since there are multiple "short-range" paths to connect long-range spin carrying units. Then a small fluctuation of the short-range alignments $\left(P_{i j}(J)\right)$ will produce a small deviation on the total energy, but a large deviation of the long-range spin correlation. In line with this argument, XANKED has the smallest $(\%$ $\left.T_{C} C_{s} / C_{p}\right)$ value, which is also compatible with its magnetic topology being a strong rung spin ladder. Since the spin-ladder has largest J(rung) interaction than $J($ rail), short-range spin correlation within a rung (i.e. between a 2 spin-carrying radicals, 2 s model) has similar behavior as a 2s quasi-isolated topology. We can thus conclude that the discrepancy between $T_{C}$ computed from magnetic $C_{s}(T)$ and heat $C_{p}(T)$ capacities can be explained in terms of the order in the crystal. It must be further emphasized that the systematic shift of the transition temperature computed from $C_{s}(T)$ and $C_{p}(T)$ (i.e. $\left.T_{C}^{\mu}\left(C_{s}\right)<T_{C}^{h}\left(C_{p}\right)\right)$ ), which was observed for the $4 \mathrm{~s}$ model, is also realized for the seven real molecule-based magnets here analyzed due to the different behavior of both short- and long-range spin correlations. Note also that, even if the difference between $T_{C}^{\mu}\left(C_{s}\right)$ and $T_{C}^{h}\left(C_{p}\right)$ critical temperatures is not very large, it can be of crucial importance when studying physical properties around the critical point. 


\section{Conclusions}

In this paper it is established that the heat capacity $C_{p}(T)$ measures the energy variation due to the 3D propagation of the interaction of two magnetically connected spins, i.e. to shortrange ordering. The magnetic capacity $C_{s}(T)$, in turn, is a measure of the thermal variation of the spin multiplicity of the system, and reflects the importance of magnetically nonconnected spin alignment and how the dominant effect of long-range spin correlation governs the magnetic behavior of molecule-based crystals (and in general of magnetic compounds).

We show that the current definition of the critical temperature $T_{C}$ for magnetic systems, which is associated with a maximum in the heat capacity $C_{p}(T)$, does not capture the magnetic nature because it excludes long-range magnetic order. Instead, a maximum in $\partial[\chi T(T)] / \partial T$, that is related to the magnetic capacity $C_{s}(T)$ which in turn includes changes in short- and long- range spin order/disorder, is a more broadly applicable definition of the magnetic transition temperature.

The magnetic capacity $C_{s}(T)$ is also useful because it can be interpreted in terms of the molecular structure of the crystal using the magnetic wavefunction for each magnetic microstate. For this interpretation, we have used a Valence Bond analysis of the magnetic wavefunction. This analysis uses the concept of the Boltzmann magnetic bond order $(\tilde{\mu}(T))$, which is in turn defined using the exchange density matrix $P_{i j}$. Whereas the lat-

ter provides information about the spin correlation between magnetic units, the Boltzmann magnetic $\mu$-bond order elements, $\tilde{\mu}(J, T)$ and $\tilde{\mu}(0, T)$, are a very powerful tool to visualize the temperature-dependence of the magnetic correlation between all spin units.

After analysis of seven experimental magnetic systems, we demonstrate the existence of a systematic shift between the transition temperatures associated with $C_{s}(T)$ and $C_{p}(T)$ which we demonstrate can be attributed to the loss of long-range spin correlation. We conclude that $T_{C}$ calculated from either $C_{p}(T)$ or $C_{s}(T)$ will be nearly equal only in those moleculebased systems in which the long-range spin correlation can be neglected, or there is no 3D propagation of the spin coupling. This suggests that the magnetic capacity $C_{s}(T)$ can be 
used as a predictive tool for the magnetic topology, and thus for the synthetic chemists. In addition, this type of analysis will enable further research to promote better understanding about what happens in the region close to the magnetic transition regime as a function of the magnetic topology.

\section{Acknowledgement}

JJS and JB thank he European Research Council (ERC-2010-AdG-267374), Spanish grant (FIS2016-79464-P), and Grupos Consolidados (IT578-13) for the financial support. JJS gratefully acknowledges the Spanish grant IJCI-2014-22204. JB also acknowledges funding from the European Unions Horizon 2020 research and innovation program under the Marie Sklodowska-Curie Grant Agreement No. 703195. MD thanks the Spanish Government for financial support (projects MAT2008-02032/MAT and MAT2014-54025-P), and the Catalan DURSI (2005-SGR-0036 and 2014-SGR-1422). MD also acknowledges AGAUR for a travel grant (2007BE200188). We also acknowledge Prof. Mark M. Turnbull for fruitful discussions.

\section{Supporting Information Available}

The following files are available free of charge.

- Detailed description of the bottom-up procedure used on the theoretical study of the magnetic properties (Section 1. Overview of the First-Principles Bottom-Up (FPBU) working strategy). Construction of a 4 electron paired Valence Bond basis set (Section 2: Paired Valence Bond function). Physical interpretation of the spin exchange density matrix elements of benzene resonant picture (Section 3: Valence Bond: Kekulé vs Dewar). Description and characterization of the multiconfigurational eigenstates of the

4s model (Section 4: Magnetic wavefunction decomposition (GS and ExS)). Boltzmann 
$\mu$-bond order study for the KAXHAS molecular magnet (Section 5: $\mu$-Bond order analysis of KAXHAS crystal).

This material is available free of charge via the Internet at http://pubs.acs.org/.

\section{Notes and References}

(1) Langevin, P. Magnetism and theory of electrons. Annales de Chimie et de Physique 1905, 5, 70-127.

(2) Langevin, P. Sur la théorie du magnétisme. Journal de Physique Théorique et Appliquée 1905, 4, 678-693.

(3) Weiss, P. L'hypothèse du champ moléculaire et la propriété ferromagnétique. J. Phys. Theor. Appl. 1907, 6, 661-690.

(4) Rosenfeld, L., Nielsen, J. R., Eds. EARLY WORK (1905-1911); Niels Bohr Collected Works; Elsevier, 1972; Vol. 1; pp 497-586.

(5) Van Vleck, J. In The International Series of Monographs on Physics; Flowler, R., Kapitza, P., Eds.; Oxford At The Clarendon Press, 1932; p 384pp.

(6) Kahn, O. Molecular Magnetism; VCH Publisher, Inc, 1993; p 380.

(7) Trinquier, G.; Suaud, N.; Guihéry, N.; Malrieu, J.-P. Designing Magnetic Organic Lattices from High-Spin Polycyclic Units. ChemPhysChem 2011, 12, 3020-3036.

(8) Mayhall, N. J.; Head-Gordon, M. Computational quantum chemistry for single Heisenberg spin couplings made simple: Just one spin flip required. Journal of Chemical Physics 2014, 141.

(9) Ivancevic, V. G.; Ivancevic, T. T. Springer Series: Understaning Complex Systems; Springer-Verlag Berlin Heidelberg, 2008; p 884. 
(10) Carlin, R. L. Magnetochemistry; Springer-Verlag Berlin Heidelberg, 1986.

(11) Fisher, M. E. Relation between the specific heat and susceptibility of an antiferromagnet. Philosophical Magazine 1962, 7, 1731-1743.

(12) Wolf, W. P. Review letters 2 1. Physical Review Letters 1964, 13, 3-5.

(13) Ru, N.; Chu, J. H.; Fisher, I. R. Magnetic properties of the charge density wave compounds RTe3, R=Y, La, Ce, Pr, Nd, Sm, Gd, Tb, Dy, Ho, Er \& Tm. Physical Review $B 2008,78,4$.

(14) Narsinga Rao, G.; Sankar, R.; Panneer Muthuselvam, I.; Chou, F. C. Magnetic and thermal property studies of $\mathrm{RCrTeO6}(\mathrm{R}=$ trivalent lanthanides) with layered honeycomb sublattices. Journal of Magnetism and Magnetic Materials 2014, 370, 13-17.

(15) Thota, S.; Singh, K.; Nayak, S.; Simon, C.; Kumar, J.; Prellier, W. The ac-magnetic susceptibility and dielectric response of complex spin ordering processes in Mn3O4. Journal of Applied Physics 2014, 116, 0-8.

(16) Bearpark, M. J.; Robb, M. A.; Bernardi, F.; Olivucci, M. Molecular mechanics valence bond methods for large active spaces. Application to conjugated polycyclic hydrocarbons. Chemical Physics Letters 1994, 217, 513-519.

(17) Pauncz, R. Spin eigenfunctions: Construction and Use; PLENUM PRESS, 1979; pp $\mathrm{XV}, 370$.

(18) White, R. M. Springer Series in SOLID-STATE SCIENCES; Springer-Verlag Berlin Heidelberg, 2007; Vol. 138; p 359.

(19) Deumal, M.; Bearpark, M. J.; Novoa, J. J.; Robb, M. A. Magnetic properties of organic molecular crystals via an algebraic Heisenberg Hamiltonian. Applications to WILVIW, TOLKEK, and KAXHAS nitronyl nitroxide crystals. Journal of Physical Chemistry A 2002, 106, 1299-1315. 
(20) McWeeny, R.; Sutcliffe, B. T. Methods of molecular quantum mechanics; Academic Press London, New York, 1969; pp ix, 307 p.

(21) Anderson, P. W. New Approach to the Theory of Superexchange. Physical Review $1959,115,1-13$.

(22) Said, M.; Maynau, D.; Malrieu, J.-P.; Garcia-Bach, M. A. A nonempirical Heisenberg Hamiltonian for the study of conjugated hydrocarbons. Ground-state conformational studies. Journal of the American Chemical Society 1984, 106, 571-579.

(23) Malrieu, J.-P.; Maynau, D. A Valence Bond Effective Hamiltonian for Neutral States of $\pi$ Systems. 1. Method. Journal of the American Chemical Society 1982, 104, 30213029.

(24) Durand, P.; Malrieu, J.-P. Advances in Chemical Physics: Ab Initio Methods in Quantum Chemistry Part I; John Wiley \& Sons, Inc., 1987; Vol. 67; pp 321-412.

(25) Malrieu, J.-P. In The Concept of the Chemical Bond: Theoretical Models of Chemical Bonding Part 2; Maksic, Z. B., Ed.; Springer-Verlag Berlin Heidelberg, 1990; pp 107136.

(26) Fumanal, M.; Deumal, M. The quest for rationalizing the magnetism in purely organic semiquinone-bridged bisdithiazolyl molecular magnets. Phys. Chem. Chem. Phys. 2016, 18, 20738-20749.

(27) Vela, S.; Deumal, M.; Shiga, M.; Novoa, J. J.; Ribas-Arino, J. Dynamical effects on the magnetic properties of dithiazolyl bistable materials. Chemical Science 2015, 23712381.

(28) Herringer, S. N.; Deumal, M.; Ribas-Arino, J.; Novoa, J. J.; Landee, C. P.; Wikaira, J. L.; Turnbull, M. M. S=1/2 one-dimensional random-exchange ferromagnetic 
zigzag ladder, which exhibits competing interactions in a critical regime. Chemistry A European Journal 2014, 20, 8355-8362.

(29) Vela, S.; Sopena, A.; Ribas-Arino, J.; Novoa, J. J.; Deumal, M. Elucidating the 2D magnetic topology of the 'metal-radical' TTTA.Cu(hfac $)_{2}$ system. Chemistry - A European Journal 2014, 20, 7083-7090.

(30) Vela, S.; Deumal, M.; Ribas-Arino, J.; Novoa, J. J. Tracing the sources of the different magnetic behavior in the two phases of the bistable (BDTA $)_{2}\left[\mathrm{Co}(\mathrm{mnt})_{2}\right]$ compound. Inorganic Chemistry 2012, 51, 8646-8648.

(31) Clarke, C. S.; Jornet-Somoza, J.; Mota, F.; Novoa, J. J.; Deumal, M. Origin of the magnetic bistability in molecule-based magnets: A first-principles bottom-up study of the TTTA crystal. Journal of the American Chemical Society 2010, 132, 17817-17830.

(32) Deumal, M.; Rawson, J. M.; Goeta, A. E.; Howard, J. A.; Copley, R. C.; Robb, M. A.; Novoa, J. J. Studying the origin of the antiferromagnetic to spin-canting transition in the $\beta$-p- $\mathrm{NCC}_{6} \mathrm{~F}_{4} \mathrm{CNSSN} \cdot$ molecular magnet. Chemistry - A European Journal 2010, $16,2741-2750$.

(33) Coulson, P. C. A.; Fischer, M. I. XXXIV. Notes on the molecular orbital treatment of the hydrogen molecule. The London, Edinburgh, and Dublin Philosophical Magazine and Journal of Science 1949, 40, 386-393.

(34) Wilson, S. In Advances in the Theory of Atomic and Molecular Systems: Conceptual and Computational Advances in Quantum Chemistry; Piecuch, P., Maruani, J., Eds, S. W., Wilson, S., Eds.; 2009; Vol. 19; pp 269-294.

(35) Bearpark, M. J.; Smith, B. R.; Bernardi, F.; Olivucci, M.; Robb, M. A. Combined Quantum Mechanical and Molecular Mechanical Methods; American Chemical Society, 1998; Vol. 712; pp 148-158 SE - 9. 
(36) Szabo, A.; Ostlund, N. S. Modern Quantum Chemistry: Introduction to Advanced Electronic Structure Theory; Dover Books on Chemistry; Dover Publications, 1989; p 480.

(37) Blancafort, L.; Celani, P.; Bearpark, M. J.; Robb, M. A. A valence-bond-based complete-active-space self-consistent-field method for the evaluation of bonding in organic molecules. Theoretical Chemistry Accounts 2003, 110, 92-99.

(38) Mcweeny, R. Spins in Chemistry; Current Chemical Concepts; Academic Press London, New York, 1970; p 153.

(39) Deumal, M.; Bearpark, M. J.; Robb, M. A.; Pontillon, Y.; Novoa, J. J. The mechanism of magnetic interactions in the bulk ferromagnet para-(methylthio)phenyl nitronyl nitroxide (YUJNEW): A first principles, bottom-up, theoretical study. Chemistry - A European Journal 2004, 10, 6422-6432.

(40) Angelini, M. C.; Parisi, G.; Ricci-Tersenghi, F. Relations between short-range and longrange Ising models. Physical Review E - Statistical, Nonlinear, and Soft Matter Physics 2014, 89, 1-11.

(41) Bera, A. K.; Yusuf, S. M.; Kumar, A.; Majumder, M.; Ghoshray, K.; Keller, L. Longrange and short-range magnetic correlations, and microscopic origin of net magnetization in the spin-1 trimer chain compound $\mathrm{CaNi}_{3} \mathrm{P}_{4} \mathrm{O}_{14}$. Physical Review B 2016, 93, $1-9$.

(42) Medvedev, M. V. Short-range magnetic order in a three-dimensional XXZ model with competing exchange interactions. The Physics of Metals and Metallography 2009, 10\%, $123-132$.

(43) Hinatsu, Y.; Doi, Y. Structural phase transition and antiferromagnetic transition of $\mathrm{Tb}$ 3RuO7. Journal of Solid State Chemistry 2014, 220, 22-27. 
(44) Aharen, T.; Greedan, J. E.; Ning, F.; Imai, T.; Michaelis, V.; Kroeker, S.; Zhou, H.; Wiebe, C. R.; Cranswick, L. M. D. Magnetic properties of the the $\mathrm{S}=3 / 2$ geomterically frustrated double perovskites $\mathrm{La}_{2} \mathrm{LiRuO}_{6}$ and $\mathrm{Ba}_{2} \mathrm{YRuO}_{6}$. Physical Review B 2009, 80, 134423.

(45) Jornet-Somoza, J.; Deumal, M.; Robb, M. A.; Landee, C. P.; Turnbul, M. M.; Feyerherm, R.; Novoa, J. J. First-principles bottom-up study of 1D to 3D magnetic transformation in the copper pyrazine dinitrate $\mathrm{S}=1 / 2$ antiferromagnetic crystal. Inorganic Chemistry 2010, 49, 1750-1760.

(46) Jornet, J.; Deumal, M.; Ribas-Ariño, J.; Bearpark, M. J.; Robb, M. A.; Hicks, R. G.; Novoa, J. J. Direct versus mediated through-space magnetic interactions: A first principles, bottom-up reinvestigation of the magnetism of the pyridyl-verdazyl:hydroquinone molecular co-crystal. Chemistry - A European Journal 2006, 12, 3995-4005.

(47) Shapiro, A.; Landee, C. P.; Turnbull, M. M.; Jornet, J.; Deumal, M.; Novoa, J. J.; Robb, M. A.; Lewis, W. Synthesis, structure, and magnetic properties of an antiferromagnetic spin-ladder complex: Bis(2,3-dimethylpyridinium) tetrabromocuprate. Journal of the American Chemical Society 2007, 129, 952-959.

(48) Jornet-Somoza, J.; Codina-Castillo, N.; Deumal, M.; Mota, F.; Novoa, J. J.; Butcher, R. T.; Turnbull, M. M.; Keith, B.; Landee, C. P.; Wikaira, J. L. Synthesis, structure, magnetic behavior, and theoretical analysis of diazine-bridged magnetic ladders: $\mathrm{Cu}$ (quinoxoline) $\mathrm{X}_{2}$ and $\mathrm{Cu}\left(2,3\right.$-dimethylpyrazine) $\mathrm{X}_{2}(\mathrm{X}=\mathrm{Cl}, \mathrm{Br})$. Inorganic Chemistry 2012, 51, 6315-6325.

(49) Vela, S.; Jornet-Somoza, J.; Turnbull, M. M.; Feyerherm, R.; Novoa, J. J.; Deumal, M. Dividing the spoils: Role of pyrazine ligands and perchlorate counterions in the magnetic properties of bis(pyrazine)diperchloratecopper(II), $\left[\mathrm{Cu}(\mathrm{pz})_{2}\right]\left(\mathrm{ClO}_{4}\right)_{2}$. Inorganic Chemistry 2013, 52, 12923-12932. 
(50) Li, X.; Turnbull, M. M.; Landee, C. P.; Jornet, J.; Deumal, M.; Novoa, J. J.; Wikaira, J. L. Synthesis, Structure, and Magnetic Behavior of Bis(2-amino-5fluoropyridinium) Tetrachlorocuprate(II). Inorganic Chemistry 2007, 46, 11254-11265.

(51) CCDC and chemical names for the studied molecular magnets: CUPYAR (pyrazine)dinitratocopper(II); TEXDEG bis(2,3-dimethylpyridinium) tetrabromocuprate(II); XANKED bis(2,3-dimethylpyrazine)dihalocopper(II); CUPYZP bis(pyrazine)diperchloratecopper(II); GIVZOB bis(2-amino-5- fluoropyridinium) tetrachlorocuprate(II); UBUBIC pyridyl-verdazyl radical:hydroquinone co-crystal; KAXHAS p-nitrophenyl nitronyl nitroxide.

(52) Jornet-Somoza, J.; Deumal, M.; Turnbull, M. M.; Novoa, J. J. On the existence of temperature induced changes in the magnetic topology of crystals that show no firstorder crystallographic phase transitions. Polyhedron 2009, 28, 1965-1971. 


\section{TOC Graphic}

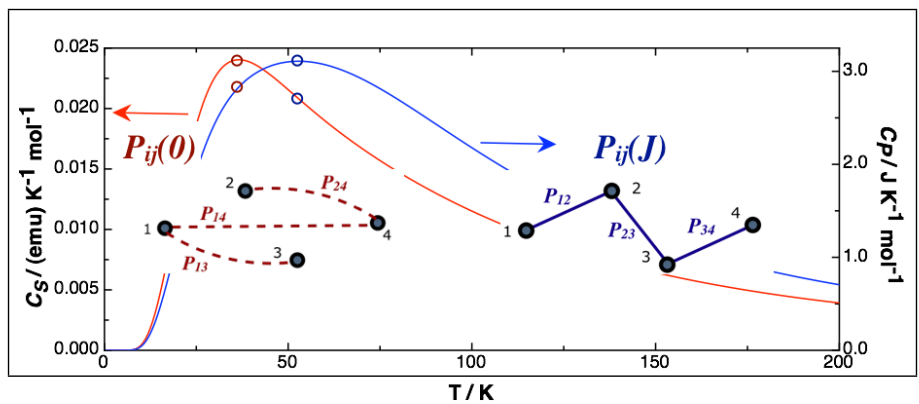

